2009-12-01

\title{
Compact Trench-Based Silicon-On-Insulator Rib Waveguide Ring Resonator With Large Free Spectral Range
}

\author{
Seunghyun Kim \\ Gregory P. Nordin \\ nordin@byu.edu \\ Weisheng $\mathrm{Hu}$ \\ Yusheng Qian \\ Jiguo Song
}

See next page for additional authors

Follow this and additional works at: https://scholarsarchive.byu.edu/facpub

Part of the Electrical and Computer Engineering Commons

\section{Original Publication Citation}

Y. Qian, S. Kim, J. Song, W. Hu, G. Wojcik, G.P. Nordin, "Compact trench-based SOI rib waveguide ring resonator with large free spectral range," Opt. Eng. 48(12), 12462, pp. 1-4 (29).

\section{BYU ScholarsArchive Citation}

Kim, Seunghyun; Nordin, Gregory P.; Hu, Weisheng; Qian, Yusheng; Song, Jiguo; and Wojcik, Gregory L., "Compact Trench-Based Silicon-On-Insulator Rib Waveguide Ring Resonator With Large Free Spectral Range" (2009). Faculty Publications. 850.

https://scholarsarchive.byu.edu/facpub/850

This Peer-Reviewed Article is brought to you for free and open access by BYU ScholarsArchive. It has been accepted for inclusion in Faculty Publications by an authorized administrator of BYU ScholarsArchive. For more information, please contact ellen_amatangelo@byu.edu. 
Authors

Seunghyun Kim, Gregory P. Nordin, Weisheng Hu, Yusheng Qian, Jiguo Song, and Gregory L. Wojcik 


\section{Compact trench-based silicon-on-insulator rib waveguide ring resonator with large free spectral range}

\author{
Yusheng Qian \\ Seunghyun Kim \\ Jiguo Song \\ Weisheng $\mathrm{Hu}$ \\ Brigham Young University \\ Electrical and Computer Engineering \\ Provo, Utah 84602
}

\author{
Gregory L. Wojcik, MEMBER SPIE \\ Innolume, Inc. \\ 3333 Bowers Avenue, Suite 190 \\ Santa Clara, California 95054
}

Gregory P. Nordin, MEMBER SPIE

Brigham Young University

Electrical and Computer Engineering

Provo, Utah 84602

E-mail: nordin@byu.edu

\begin{abstract}
We demonstrate a compact trench-based silicon-on-insulator (SOI) rib waveguide ring resonator comprised of trench-based bends and splitters. It has a perimeter of $50 \mu \mathrm{m}$ and occupies an area of only $25 \times 25 \mu \mathrm{m}$. The measured free spectral range (FSR) is $13.2 \mathrm{~nm}$, which the largest reported for an SOI rib waveguide ring resonator. The measured FSR, full width at half maximum, and quality factor match reasonably well with analytical calculations. Further calculation shows that a FSR of $50.8 \mathrm{~nm}$ is achievable for an SOI rib waveguide ring resonator with a perimeter of $15 \mu \mathrm{m}$. () 2009 Society of Photo-Optical Instrumentation Engineers. [DOI: 10.1117/1.3274999]
\end{abstract}

Subject terms: photonic integrated circuits; silicon-on-insulator; rib waveguide; ring resonator.

Paper 090615R received Aug. 13, 2009; revised manuscript received Oct. 20, 2009; accepted for publication Oct. 21, 2009; published online Dec. 28, 2009.

\section{Introduction}

Silicon-on-insulator (SOI) rib waveguides have been widely used as a platform for various active and passive photonic devices, ${ }^{1-9}$ and as a photonic biological sensor. ${ }^{10}$ Advantages of a SOI rib waveguide over a SOI wire waveguide are lower propagation loss, lower coupling loss from/to fiber, larger fabrication tolerance, and straightforward configuration of lateral $p-i-n$ structure for active photonic devices. However, the large radius of curvature required for waveguide bends and splitters is a drawback of SOI rib waveguides, which significantly limits the degree of possible integration. Conventional ring resonators (RRs), which have received much attention for many photonic devices, are configured with two straight waveguides and either a circular or racetrack-type ring waveguide between them. Due to the large radius of curvature for a waveguide bend, the perimeter of a ring waveguide for a conventional SOI rib waveguide-based RR is typically a few hundred microns. ${ }^{7,8}$ Such a large perimeter ring not only increases the RR size but also significantly reduces the achievable free spectral range (FSR), which is one of the important characteristics of a RR. Other important parameters include the full width at half maximum (FWHM), quality (Q) factor, extinction ratio (ER), and insertion loss. Typically reported FSR values for conventional SOI rib waveguide RRs are less than $1 \mathrm{~nm}$, while the largest FSR is $3 \mathrm{~nm} .{ }^{5-9}$ Large FSR is especially important for optical filters in wavelength division multiplexing (WDM) communication systems. ${ }^{11,12}$

We have recently demonstrated compact trench-based bends (TBBs) and trench-based splitters (TBSs) for SOI rib

0091-3286/2009/\$25.00 @ 2009 SPIE waveguides, ${ }^{13,14}$ the size of which depends only on the lateral extent of the waveguide mode and not on the core/clad refractive index contrast. In this work, we experimentally demonstrate a trench-based RR (TBRR) for SOI rib waveguides by combining TBBs and TBSs. A TBRR with a perimeter of only $50 \mu \mathrm{m}$ is designed, fabricated, and measured. The measured FSR is $13.2 \mathrm{~nm}$, which, to the best of our knowledge, is the largest ever reported for an SOI rib waveguide RR.

\section{Design}

Schematic illustrations of a TBB and TBS are shown in Fig. 1(a), along with the inset illustrating the cross section of SOI rib waveguides considered in this work. The waveguide supports only the fundamental TE polarization (electric field in the plane) mode at a wavelength of $1550 \mathrm{~nm}$. Our ring resonator design and measurement are therefore performed only for TE polarization. For a TBB, the measured bend efficiency as reported in Ref. 13 is $93 \%$. Figure 1(b) shows results for a 3-D finite difference time domain (FDTD) simulation of a TBS. As the trench width increases, the splitting ratio (reflection/transmission) becomes larger, while the overall loss is increased. With a trench width of $119 \mathrm{~nm}$, the splitting ratio is 4 and the total TBS efficiency is $90 \%$.

By combining two TBBs and two TBSs, a TBRR can be realized as shown in Fig. 1(c). For a given input source, the optical power spectrum at the drop and throughput ports depend on the resonance characteristics of the ring waveguide. The optical power spectrum at the drop $\left(\eta_{\text {drop }}\right)$ and throughput $\left(\eta_{\text {throughput }}\right)$ ports can be analytically calculated $^{15}$ as 


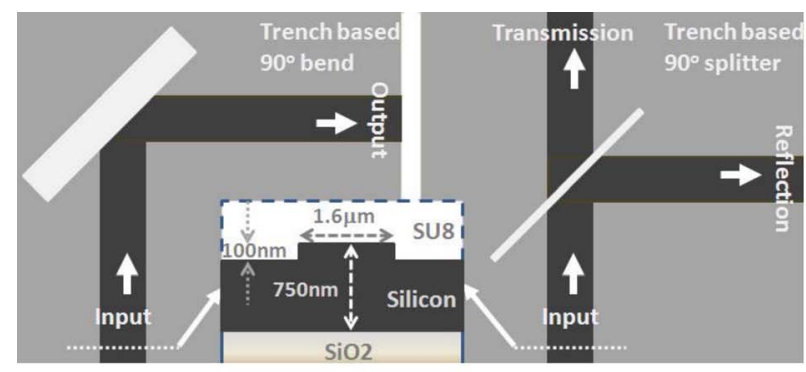

(a)

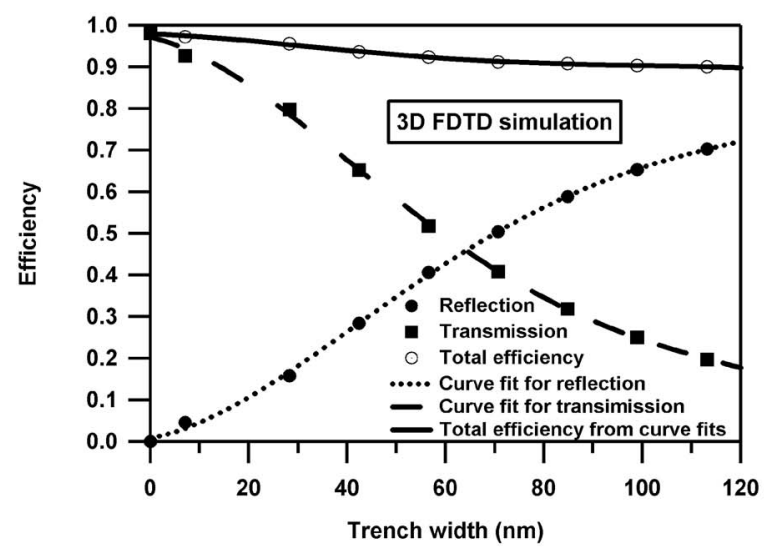

(b)

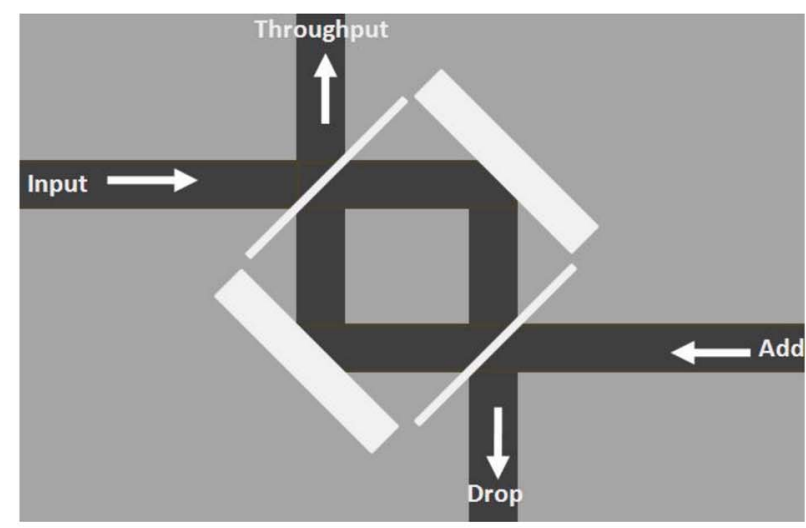

(c)

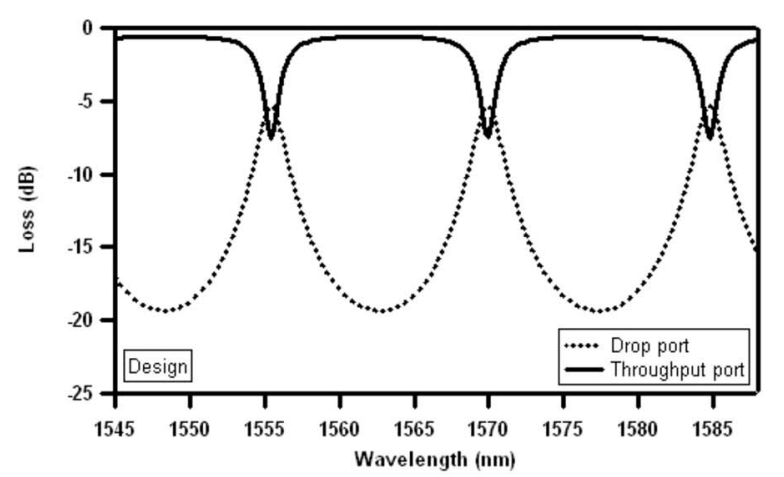

(d)

Fig. 1 (a) Schematic illustration of TBB and TBS (inset: cross section of SOI rib waveguide). (b) 3-D FDTD simulation results for a TBS with a SU8 filled trench. (c) TBRR realized by combining TBBs and TBSs. (d) Analytical calculation results for a TBRR.

$$
\begin{aligned}
& \eta_{\text {drop }}=\frac{T_{s}^{2}}{1+R_{s}^{2} R_{b}^{2}-2 R_{s} R_{b} \cos \delta}, \\
& \eta_{\text {throughput }}=R_{s}\left(1+\frac{T_{s}^{2} R_{b}^{2}+2 T_{s} R_{s} R_{b}^{2}-2 T_{s} R_{b} \cos \delta}{1+R_{s}^{2} R_{b}^{2}-2 R_{s} R_{b} \cos \delta}\right),
\end{aligned}
$$

where $R_{b}$ is the bend efficiency, $T_{s}$ and $R_{s}$ are the splitter transmission and reflection, $\delta=2 \pi n_{e f f} d / \lambda+\phi_{o}, n_{\text {eff }}$ is the effective refractive index of the waveguide mode, $d$ is the perimeter of a ring, and $\phi_{o}$ is the phase delay from TBBs and TBSs. Calculations for a perimeter of $50 \mu \mathrm{m}(12.5 \mu \mathrm{m}$ length on each side), $93 \%$ bend efficiency, and $90 \%$ splitter efficiency with a splitting ratio of 4 are shown in Fig. 1(d). The effective index of our rib waveguide structure calculated by FIMMWAVE from Photon Design (Oxford, United Kingdom) is 3.36, and phase delays at TBBs and TBSs are not considered in this calculation $\left(\phi_{o}=0\right)$. The calculated TBRR loss is $0.6 \mathrm{~dB}$ and the FSR is $14.7 \mathrm{~nm}$.

\section{Fabrication and Measurements}

We fabricated the structure using a process nearly the same as reported in Refs. 13 and 14. Electron beam lithography (EBL) is used to pattern the trenches for TBBs and TBSs to ensure position accuracy and smooth sidewalls. A highaspect-ratio etch is done with an inductively coupled plasma reactive ion etcher (ICP RIE) using a fluorine-based etch chemistry. At the end of the process, SU8 is spincoated to fill the trenches and also acts as an upper clad. Figure 2(a) shows a scanning electron microscope (SEM) image of a fabricated TBRR before SU8 coating. The TBB trench width is $2 \mu \mathrm{m}$, while the TBS trench width is designed to be $119 \mathrm{~nm}$. The perimeter of the fabricated TBRR is $50 \mu \mathrm{m}$. It occupies an area of only $25 \times 25 \mu \mathrm{m}$.

To characterize the fabricated TBRR, a polarized broadband superluminescent light emitting diode (SLED) is coupled to a polarization maintaining (PM) fiber, and light from the PM fiber is butt-coupled to an input SOI rib waveguide. The optical power spectrum of the SLED is measured with an optical spectrum analyzer (OSA, Ando AQ6317) used as a reference spectrum. Light from the drop and throughput ports is then sequentially butt-coupled to a single mode fiber and connected to the OSA. Figure 2(b) shows the measurement results normalized by the source spectrum. The measured insertion loss is $\sim 29 \mathrm{~dB}$, which includes coupling losses from the PM fiber to an SOI rib waveguide and from an SOI rib waveguide to a single mode fiber, propagation loss, loss from additional 90-deg bends to reroute the outputs, and loss from TBRR itself.

Table 1 shows a comparison of measurement and analytical calculation results [Fig. 1(d)] for the drop wavelengths, FSR, FWHM, Q factor, and ERs at the drop and throughput ports. The measured drop wavelengths are slightly different from the analytical calculation, because the average measured FSR, $13.15 \mathrm{~nm}$, is $1.65 \mathrm{~nm}$ smaller than predicted by analytical calculation. The difference may be due to phase delays at the TBBs and TBSs, which are not considered in the analytical calculation, and the actual effective index of the waveguide mode may be larger than predicted by FIMMWAVE. The FWHM and Q factor from measurement at the drop wavelengths of 1570.1 and 


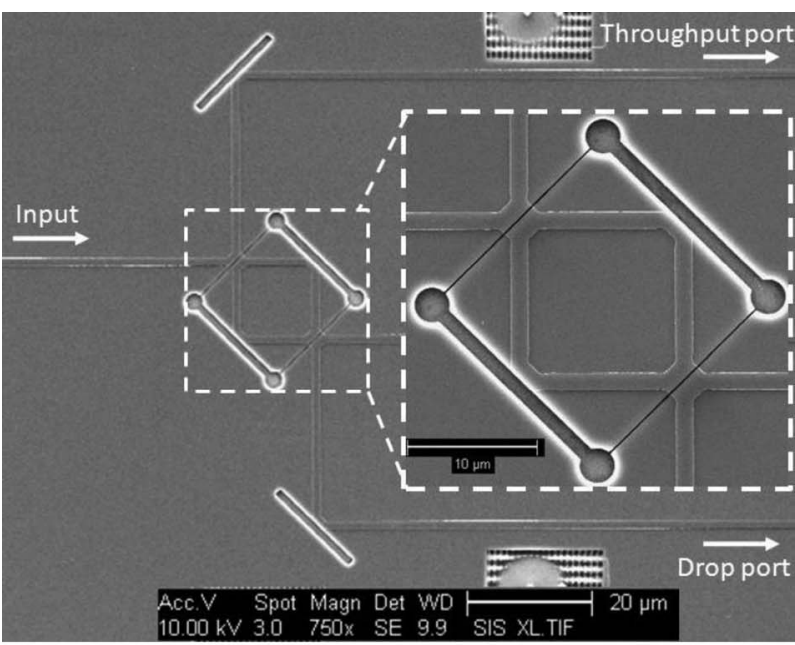

(a)

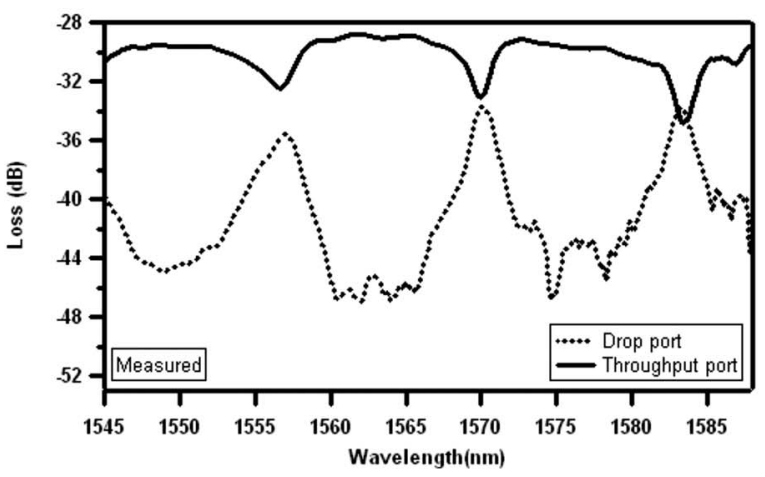

(b)

Fig. 2 (a) SEM image of fabricated TBRR (inset: close-up of TBRR region), and (b) measured TBRR drop and throughput port spectra.

$1583.3 \mathrm{~nm}$ are well matched with the analytical calculation, while the measured signal at $1557 \mathrm{~nm}$ is wider than the analytical calculation. We do not yet understand the reason for widening at this wavelength, although it may in part be due to overlap of the resonant peak and observed ripples in the spectrum. Measured ERs for both the drop and throughput ports are smaller than the calculation predicts. The reason is likely that the TBB and TBS losses are larger than

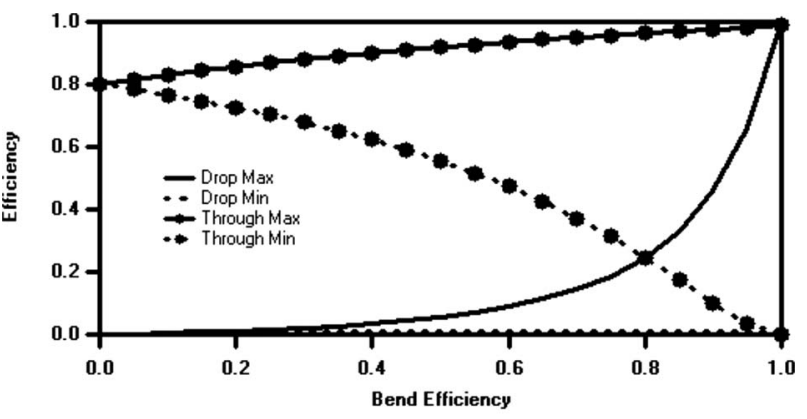

(a)

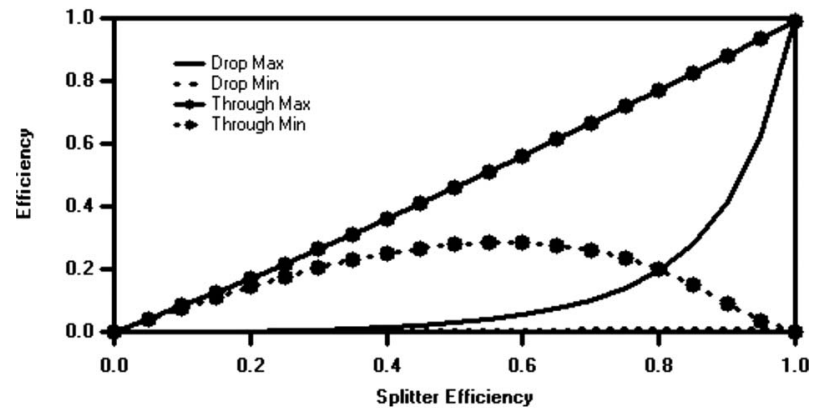

(b)

Fig. 3 Analytical calculation of maximum and minimum efficiencies at the drop and throughput ports as a function of (a) bend efficiency (assuming splitter efficiency=1), and (b) splitter efficiency (assuming bend efficiency=1).

assumed for the calculations. For example, TBB and TBS efficiencies of $81 \%$ (0.92-dB loss) and $80 \%$ (0.97-dB loss) result in an analytically calculated ER within $10 \%$ of the average measured ER. Figure 3 shows the maximum and minimum efficiencies at the drop and throughput ports as a function of bend [Fig. 3(a)] and splitter [Fig. 3(b)] efficiency. As either the bend or splitter efficiency decreases, the difference between the maximum and minimum efficiencies at both the drop and throughput ports becomes smaller, such that the ER is reduced. Note that the ERs and the TBRR loss are both more sensitive to the splitter efficiency than the bend efficiency.

Since the FSR and Q factor from the analytical calculations match reasonably well with the measurement results,

Table 1 Comparison of measurement and analytical calculation results.

\begin{tabular}{|c|c|c|c|c|c|c|c|c|c|c|}
\hline \multirow[b]{2}{*}{ Drop wavelength (nm) } & \multicolumn{5}{|c|}{ Analytical calculation } & \multicolumn{5}{|c|}{ Measurements } \\
\hline & 1555.4 & & 1570 & & 1584.8 & 1557 & & 1570.1 & & 1583.3 \\
\hline $\mathrm{FSR}(\mathrm{nm})$ & & 14.6 & & 14.8 & & & 13.1 & & 13.2 & \\
\hline FWHM (nm) & 1.86 & & 1.91 & & 1.93 & 3.31 & & 1.96 & & 2.14 \\
\hline$Q$ factor & 836.2 & & 822 & & 821.1 & 470.4 & & 801.1 & & 739.9 \\
\hline Drop port ER (dB) & 14.07 & & 14.06 & & 14.06 & 9.28 & & 9.28 & & 8.97 \\
\hline $\begin{array}{l}\text { Throughput port ER } \\
\text { (dB) }\end{array}$ & 6.96 & & 6.92 & & 6.94 & 2.86 & & 3.31 & & 5.07 \\
\hline
\end{tabular}




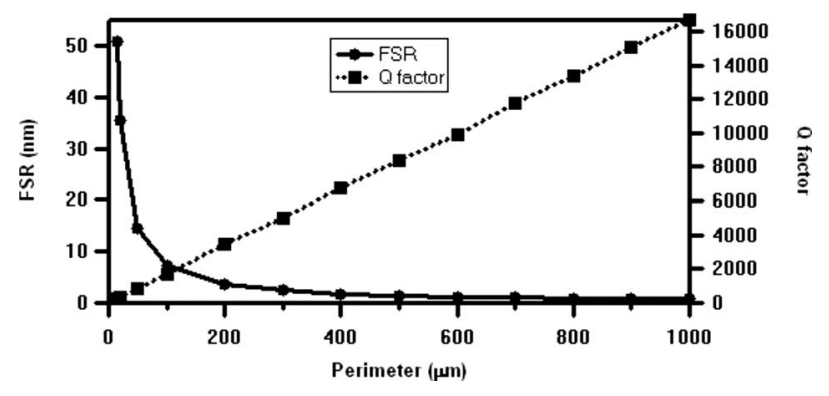

Fig. 4 FSR and Q factor as a function of perimeter for wavelengths close to $1550 \mathrm{~nm}$.

we use analytical calculation to predict the performance of smaller TBRRs. The minimum perimeter can be as small as $15 \mu \mathrm{m}$ (3.75- $\mu \mathrm{m}$ length on each side) without additional loss, because the trenches for both the TBBs and TBSs intersect more than $99 \%$ optical power in the SOI rib waveguide mode. Figure 4 shows the analytical calculation of FSR and Q factor as a function of perimeter. At a perimeter of $15 \mu \mathrm{m}$, the FSR is $50.8 \mathrm{~nm}$, while the Q factor is 246.1 . Note that a large Q factor can be achieved with a large perimeter at the cost of a smaller FSR. One way to increase the Q factor while keeping the FSR large is to create a cascaded TBRR similar to the work reported by Timotijevic et al. ${ }^{6}$

\section{Summary}

We demonstrate a compact TBRR for SOI rib waveguides. For a perimeter of $50 \mu \mathrm{m}$, the TBRR occupies an area of only $25 \times 25 \mu \mathrm{m}$, and the measured FSR is as large as $13.2 \mathrm{~nm}$, which is the smallest SOI rib waveguide RR and the largest FSR reported in the literature. The FSR, FWHM, and Q factor measurements match reasonably well with the analytical calculation, while the measured ERs at both the drop and throughput ports are smaller because of excessive loss in the fabricated TBBs and TBSs. Further fine tuning of the trench etch process should result in lower loss TBBs and TBSs. Based on analytical calculation, we show that a FSR of $50.8 \mathrm{~nm}$ is achievable for a RR with a perimeter of $15 \mu \mathrm{m}$.

\section{Acknowledgment}

This work was supported in part by Innolume, Incorporated, and DARPA grant N66001-04-8933.

\section{References}

1. G. T. Reed and A. P. Knights, Silicon Photonics: an Introduction, John Wiley and Sons Ltd., New York (2004).

2. D. Marris-Morini, L. Vivien, J. M. Fédéli, E. Cassan, P. Lyan, and S. Laval, "Low loss and high speed silicon optical modulator based on a lateral carrier depletion structure," Opt. Express 16, 334-339 (2008), see http://www.opticsinfobase.org/oe/abstract.cfm?URI=oe-16-1334.

3. W. Mathlouthi, H. Rong, and M. Paniccia, "Characterization of efficient wavelength conversion by four-wave mixing in sub-micron silicon waveguides," Opt. Express 16, 16735-16745 (2008), see http:// www.opticsinfobase.org/oe/abstract.cfm?URI=oe-16-21-16735.

4. H. Zhou, Y. Zhao, W. Wang, J. Yang, M. Wang, and X. Jiang, "Performance influence of carrier absorption to the Mach-Zehnderinterference based silicon optical switches," Opt. Express 17, 70437051 (2009), see http://www.opticsinfobase.org/abstract.cfm?URI $=$ oe-17-9-7043.

5. I. Kiyat, A. Aydinli, and N. Dagli, "Polarization characteristics of compact SOI rib waveguide racetrack resonators," IEEE Photon. Technol. Lett. 17, 2098-2100 (2005).

6. B. D. Timotijevic, F. Y. Gardes, W. R. Headley, G. T. Reed, M. J. Paniccia, O. Cohen, D. Hak, and G. Z. Masanovic, "Multi-stage racetrack resonator filters in silicon-on-insulator," J. Opt. A, Pure Appl. Opt. 8, S473-S476 (2006).

7. H. Rong, Y. Kuo, S. Xu, A. Liu, R. Jones, M. Paniccia, O. Cohen, and O. Raday, "Monolithic integrated Raman silicon laser," Opt. Express 14, 6705-6712 (2006), see http://www.opticsinfobase.org/ abstract.cfm?URI=oe-14-15-6705.

8. I. Kiyat, A. Aydinli, and N. Dagli, "Low-power thermooptical tuning of SOI resonator switch," Res. Higher Educ. 18, 364-366 (2006).

9. Q. Huang, Y. Yu, and J. Yu, "Experimental investigation on submicron rib waveguide microring/racetrack resonators in silicon-oninsulator," Opt. Commun., 282, 22-26 (2009).

10. J. W. Noh, R. Anderson, S. Kim, J. Cardenas, and G. P. Nordin, "In-plane photonic transduction of silicon-on-insulator microcantilevers," Opt. Express 16, 12114-12123 (2008), see http:// www.opticsinfobase.org/abstract.cfm?URI=oe-16-16-12114.

11. R. Grover, V. Van, T. A. Ibrahim, P. P. Absil, L. C. Calhoun, and F. G. Johnson, "Parallel-cascaded semiconductor microring resonators for high-order and wide-FSR filters," J. Lightwave Technol. 20, 900-905 (2002).

12. M. S. Nawrocka, T. Liu, X. Wang, and R. R. Panepucci, "Tunable silicon microring resonator with wide free spectral range," Appl. Phys. Lett. 89, 071110 (2006).

13. Y. Qian, S. Kim, J. Song, and G. P. Nordin, "Compact and low loss silicon-on-insulator rib waveguide $90^{\circ}$ bend," Opt. Express 14, 6020 6028 (2006), see http://www.opticsinfobase.org/oe/abstract.cfm?URI $=$ oe-14-13-6020

14. Y. Qian, J. Song, S. Kim, and G. P. Nordin, "Compact $90^{\circ}$ trenchbased splitter for silicon-on-insulator rib waveguides," Opt. Express 15, 16712-16718 (2007), see http://www.opticsinfobase.org/oe/ abstract.cfm?URI=oe-15-25-16712.

15. S. Kim, J. Cai, J. Jiang, and G. P. Nordin, "New ring resonator configuration using hybrid photonic crystal and conventional waveguide structures," Opt. Express 12, 2356-2364 (2004), see http:// www.opticsinfobase.org/oe/abstract.cfm?URI $=$ oe-12-11-2356.

Biographies and photographs of authors not availavle. 